\title{
A demodulation method of Temperature-Spectrum width based on Chirped fiber grating*
}

\author{
Si Chen ${ }^{1}$, Xiaogang chen ${ }^{1}$, Zhihui Luo ${ }^{1, a}$ and Desuo Cai ${ }^{1,2}$ \\ ${ }^{1}$ Colleges of Science, China Three Gorges Univ. Yichang 443002, China \\ ${ }^{2}$ Guangxi Water \& Power Design Institute,Nanning, GuangXi, 530023, China
}

\begin{abstract}
Based on spectral reflection characteristics of chirped FBGs, a demodulation method for complex temperature fields is proposed in this paper. Relationships between the reflective spectrums and the temperatures are investigated through theoretical analyses. Testing results are acquired from a FBG sensing model which is designed according to the newly-developed method. A simulation to get the relationships between reflective spectrums and temperatures is set which shows that this demodulation method is feasible and its demodulation precision is beyond $+/-1^{\circ} \mathrm{C}$. The method provides a new method for analyzing complex temperature fields.
\end{abstract}

\section{Introduction}

FBG sensors are widely used to monitor the safety of petro chemistry industry, bridges and large equipments owing to their considerable advantage such as antiinterference, wavelength encoding and so on. The key physical parameters of the FBGs monitored are temperature. When the temperature field on FBGs changes, the grating period will change because of coaction from the temperature expansion effect, the thermo-optic effect and the photoelastic effect resulted from internal thermal strains, and arises from a corresponding shift of the central Bragg wavelength. How to detect the Bragg tiny wavelength-shift is a major technology for FBG sensor in engineering projects. Many conventional strategies such as edge-wave based on tunable laser, tunable Fabry-Derot filter or unbalance Mach-Zehnder interference have been proposed ${ }^{[1][2]}$. However, above methods assume that FBG was loaded under uniform temperature field, and the demodulator should lock wavelength-shift accurately and process optical signal. Sensing systems based on above methods become complex, because of having some loss of accuracy for measuring imperfect temperature fields. In this paper, a novel demodulation method for complex temperature fields is proposed, a spectral reflection characteristic of chirped FBGs is analyzed intensively, an FBG sensing model using professional software is designed and this demodulation scheme by numerical simulation is verified.

\section{Principle of the Temperature-spectrum Width demodulation}

\subsection{Theories analysis}

\footnotetext{
a Corresponding author: zhihui_luo@126.com
}

In order to make the refractive index of fiber core higher than in cladding, the metal elements such as germanium (Ge) mixed in the fiber core and the cladding is pure quartz. Therefore the fiber core possesses photosensitive properties.

Usually, the period of uniform FBG is a constant. When FBG is loaded under complex temperature field ${ }^{[3]}$ [4], gratings in different positions inside it will change in a varying manner, changing it into a chirped FBG (CFBG)

[5]. Compared with the bandwidth of different pitchs, the gap between their reflective spectrums is smaller, so all spectrums will overlap with each other to form a whole broadening reflective spectrum ${ }^{[6][7][8]}$. The increment of the whole reflective spectrum is $\Delta \lambda_{B W}$, which is the difference between the maximal increment $\Delta \lambda_{\max }$ and the minimum increment $\Delta \lambda_{\text {min }}$ of resonant wavelength in different pitchs ${ }^{[9][10][11]}$ :

$\Delta \lambda_{B W}=\Delta \lambda_{\max }-\Delta \lambda_{\min }$

Given that uniform temperature field is loaded without strain resulted from external forces, taking no account of the effect of thermal expansion and other physical effects, only axial temperature changes grating pitchs and leads the shift of Bragg wavelength, so of the shift value may be given bellow ${ }^{[6]}$.

$$
\Delta \lambda_{B}=2\left[\frac{\partial n_{e f f}}{\partial T} \Delta T+\left(\Delta n_{e f f}\right)_{e p}+\frac{\partial n_{e f f}}{\partial a} \Delta a\right] \Lambda+2 n_{e f f} \frac{\partial \Lambda}{\partial T} \Delta T
$$

where $n_{\text {eff }}$ is the effective index of refraction of the core of single mode fiber(SMF); $\Delta T$ is the difference of temperatures in different positions; $\Delta a$ is the increment 
of fiber core diameter; $\left(1 / n_{\text {eff }}\right) \frac{\partial n_{\text {eff }}}{\partial T}$ is the thermo-optic coefficient $\alpha_{n}$ of SMF; $\left(\Delta n_{e f f}\right)_{e p}$ is the photoelastic coefficient resulted from thermal expansion; $\frac{\partial n_{\text {eff }}}{\partial a}$ is the waveguide effect resulted from core diameter change induced by thermal expansion; $\frac{1}{\Lambda} \frac{\partial \Lambda}{\partial T}$ is the linear coefficient $\alpha_{\Lambda}$ of thermal expansion. Formula (2) may be simplified as follows:

$$
\frac{\Delta \lambda_{B}}{\lambda_{B} \Delta T}=\frac{1}{n_{e f f}}\left[n_{e f f} \alpha_{n}+\frac{n_{e f f}}{\Delta T}\left(\Delta n_{e f f}\right)_{e p}+\frac{\partial n_{e f f}}{\partial a} \frac{\Delta a}{\Delta T}\right]+\alpha_{\Lambda}
$$

Given that SMF is an isotropy structure, obtain the increment of FBG's bandwidth resulted from both the photoelastic effect and the waveguide effect by analyzing strain sensing model ${ }^{[6]}$,Formula (4) of the temperature sensibility coefficient is deduced as follows:

$\frac{\Delta \lambda_{B}}{\Delta T}=\left\{\frac{1}{n_{e f f}}\left[n_{e f f} \alpha_{n}-\frac{n_{e f f}^{3}}{2}\left(P_{11}+2 P_{12}\right) \alpha_{\Lambda}+S_{v g} \frac{\Delta a}{\Delta T}\right]+\alpha_{\Lambda}\right\} \lambda_{B}$

where $S_{w g}$ is the shift coefficient of Bragg wavelength by the waveguide effect. When the material of SMF is given, the increment of FBG's bandwidth is correlated with the temperature coefficient and the material coefficient. For $\mathrm{SiO} 2$ fiber, $S_{w g}$ is out of account, $\alpha_{n}$ is $8.6 \times 10^{-6} /{ }^{\circ} \mathrm{C}, \alpha_{\Lambda}$ and is $5.5 \times 10^{-7} /{ }^{\circ} C$. When the central wavelength of FBG is $1.55 \mathrm{um}$, the ratio of $\Delta \lambda_{B}$ to $\Delta T$ is $10.8 \mathrm{pm} /{ }^{\circ} \mathrm{C}$ theoretically. When a uniform FBG is loaded by different temperatures along axial direction, according to Formula (4), the increment on the whole bandwidth is proportional to the difference between increments of both ends of FBG. So Formula (5) is given bellow:

$$
\Delta \lambda_{B W}=\Delta \lambda_{B 2}-\Delta \lambda_{B 1} \approx 10.8\left(\Delta T_{\text {max }}-\Delta T_{\text {min }}\right)
$$

For nonuniform temperature fields such as linear increment fields or Gaussian fields, if variations of the temperature on FBG are continuous, Formula (5) is universal. But the coefficient of 10.8 should be modified according to specific temperature fields and parameters of FBGs. The following discussion will focus on nonuniform linear field, and at the same time given that the temperature at the start point of FBG is a basic value and increases along the axial of FBGs gradually ${ }^{[4][5]}$.

\section{2 the system model}

According to the above discussion, there is a linear relationship between the increment of the reflective spectrum width and the temperature difference on both ends of FBG, theoretically. The key point of the demodulation of the temperature is how to measure the increment. In related papers ${ }^{[1]}{ }^{[2]}$ some methods such as complex circuits or optical interference are discussed to detect the increment of the spectrum width, but they are difficult to implement. In order to simplify the spectrum width measuring, the sensor system is designed as Figure 1. Choosing the tunable SOA laser with the narrow spectrum as the optical source, the narrow optical pulses from the source pass through the optical circulator and launch into SMF. After arriving at the FBG, pulses are reflected within the range of the FBG reflective spectrum and the others transmit away. Reflective pulses transmit backward in the same SMF and pass through the circulator into the APD detector, and they are converted into electronical signals. The control device makes a high-speed decision according to the signal power and counts the number of qualified signals, and sends control signals to the pulse generator so that the laser is adjusted in time. At the same time, the control device shares datum with the connected computer which displays the number of qualified pulses and calculates the spectrum width by a given method. Usually, calibrating the sensor should be considered at first. Then calculating the temperature value according to Formula (5), the demodulation of the temperature is fulfilled.

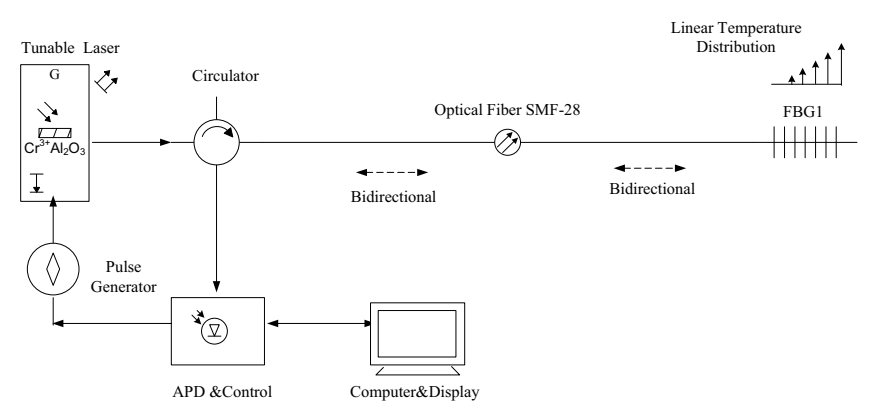

Figure 1. Schematic diagram of FBG sensing system

\section{The simulation design}

OptiGating is a professional software based on Coupled Mode Equations to design and simulation FBG. Design the uniform FBG using OptiGraing as follows: the central wavelength is $1550 \mathrm{~nm}$, the radius of the core/cladding is $4.15 \mathrm{um} / 62.5 \mathrm{um}$ and their refractive indices are 1.46/1.45, the refraction index modulation depth is 0.0005 , the period is $0.5138 \mathrm{um}$, the refraction of gate region conforms with sinusoidal distribution and the total length is $4.8 \mathrm{~cm}^{[12][13][14]}$. Using the embedded sensor module in OptiGrating, we set the thermo-optic coefficient $\alpha_{n}$ to be $8.6 \times 10^{-6} /{ }^{\circ} \mathrm{C}$ and the linear expansion coefficient $\alpha_{\Lambda} \quad 5.5 \times 10^{-7} /{ }^{\circ} \mathrm{C}$, and the ambient temperature $25^{\circ} \mathrm{C}$. The temperature field is distributed linearly along the axial, and then a FBG model which may be dragged freely is obtained. Its physic effects are simulated more closely using the high-capacity numerical calculation. Create the sensing platform of temperature-spectrum width in OptiSystem, a professional optical communication software, as Figure 1 and transplant the FBG model from OptiGrating to build a virtual FBG 
sensing system as Figure 2. We set the output power of the $\mathrm{cw}$ laser with iterative sweeping function to $0 \mathrm{dbm}$ and the bandwidth to $10 \mathrm{MHz}$, and the number of sweeping steps according to specific sites. We choose G.652 SMF to transmit the optical signal and set its loss coefficient to $0.2 \mathrm{~dB} / \mathrm{km}$ and the length to $0.5 \mathrm{~km}$. After arriving at $\mathrm{FBG}$, the optical signal within spectrum width was reflected and it transmits $0.5 \mathrm{~km}$ into detector ADP. In order to collect real-time datum easily and observe directly, we use the virtual instruments such as optical power meter, optical spectrum analyzer, oscilloscope visualize and so on which are found in the OptiSystem toolbox that performs signal detecting and extracting the power and optical spectrums.

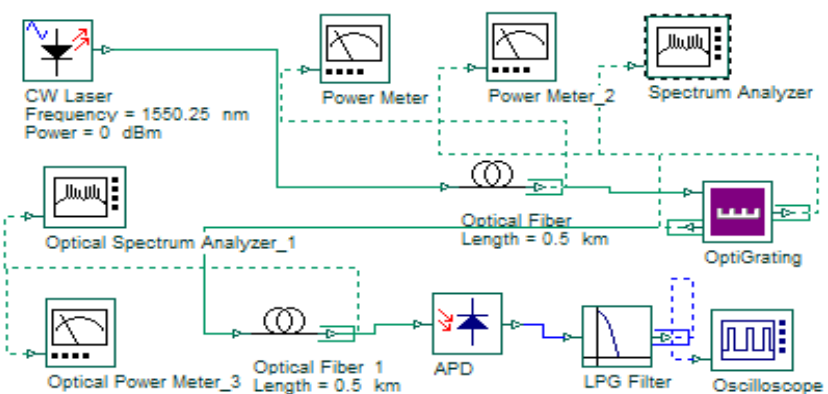

Figure 2. Model of FBG sensing system

Set parameters of the laser as follows: the tunable range of wavelength ranges from $1549.505 \mathrm{~nm}$ to $1550.50 \mathrm{~nm}$ with the step scanning of $0.005 \mathrm{~nm}$, and the linear increment of temperature is $1^{\circ} \mathrm{C}$ from $25^{\circ} \mathrm{C}$ to $35^{\circ} \mathrm{C}$. Run the simulation and get the output spectrum of APD as Figure 3 (which only shows the spectrogram at $25^{\circ} \mathrm{C}$ and $35^{\circ} \mathrm{C}$ ), the number of pulses whose power are among Full Wave at Half Maximum (FWHM)of the pulse envelope is $15 、 17 、 18 、 20 、 22 、 24 、 27 、 29$ 、 32, 35 and 37 respectively.

During simulation, the reflective spectrum broadens with the increase of temperature, and at the same time the central wavelength of FBG shifts. Moreover, both edges of spectrums fluctuate randomly as a result of laser side lobes and optical line bandwidth, and this instability will lead to some error during counting qualified output pulses. In order to make the reflective spectrums homogenized, the new technology of tan apodization method is adopted to suppress laser side lobes effect and improve the measuring accuracy. Figure 4 is reflective spectrums of apodized FBG, and all edges become steady. We run simulation and count the number of qualified pulses as follows: $11,12 、 13 、 14 、 16 、 17,18 、 21 、 23$ 、 25, 27 .

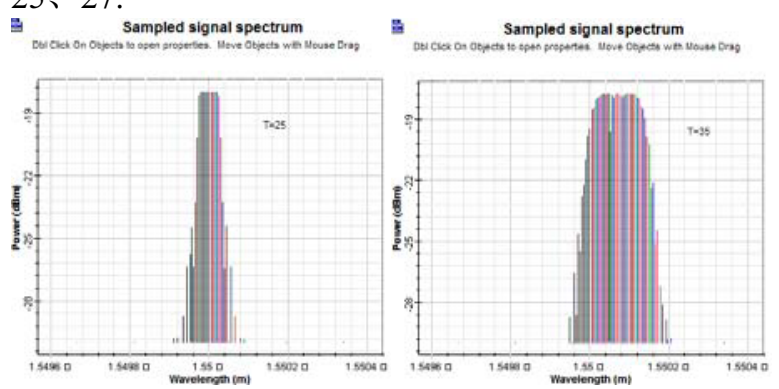

Figure 3. Spectrums of FBGs at $25^{\circ} \mathrm{C} 、 35^{\circ} \mathrm{C}$

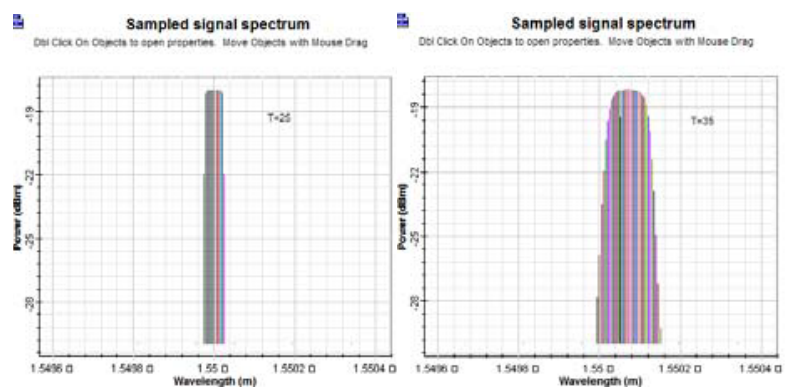

Figure 4. Spectrums of apodized FBG in $25^{\circ} \mathrm{C} 、 35^{\circ} \mathrm{C}$

Analyzing two sets of datum, we draw curves of the temperature-pulse as Figure 5. Graients of two curves are different, one is 11.2(Curve 1) and the other is 7.91(Curve 2) respectively. Because the Curve 2 is from datum of apodized FBG, fit Curve 1 using Origin software firstly and get the linear relationship of temperature and pulse number as follows:

$\Delta \lambda_{\text {FWHM }} \approx 11.2 \Delta \mathrm{T}$

The coefficient error of simulated curves is 0.389 , and the measure of its relevancy is 0.994 . Because the minimum increment of temperature is set as $1{ }^{\circ} \mathrm{C}$ and the counting error is $+/-1$, the error of spectrum width from the detector is $+/-5 \mathrm{pm}$ and the error of the whole demodulation is less than $+/-1{ }^{\circ} \mathrm{C}$. Decreasing the sweeping space between steps of the laser to $1 \mathrm{pm}$, the accuracy of FBGs will be improved effectively.

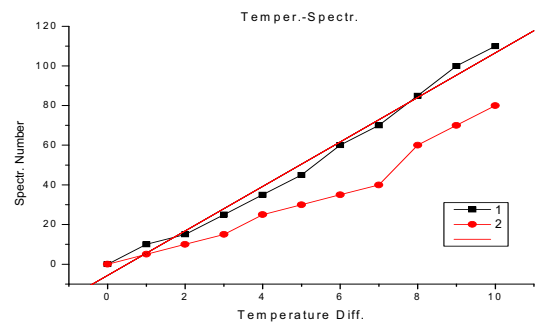

Figure 5. Curves of temperature-pulse

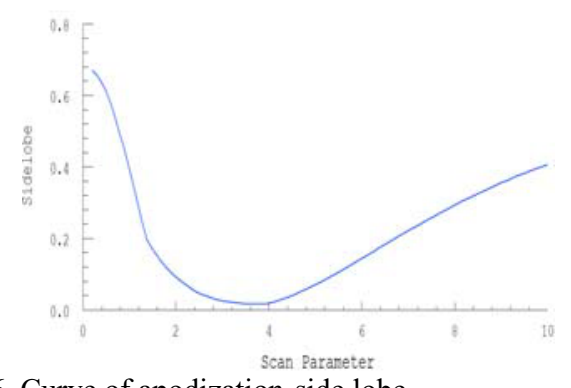

Figure 6. Curve of apodization-side lobe

\section{Results and discussion}

According to the above analysis, under the linearly increasing nonuniform temperature field, there is a linear relationship between the temperature and the increment of spectrum width, and the linear coefficient is 11.2 which is close to the theoretical value of 10.8, these data demonstrate the validity of the Formula. The coefficient error of 0.4 results from noises of reflective spectrums 
and curve fitting. Some measures such as apodizing, decreasing the space between sweeping steps and optimizing FBG are helpful to improve the demodulation accuracy. Figure 6 is the curve of apodization-side lobe which scans the apodization value from 0.1 to 10 by simulation. When the apodization value is 3.5 , the suppression of the undesirable modes is optimal ${ }^{[15]}$. But nonuniform temperature field will displace the centre wavelength of FBG and weaken the adopization effect. From Figure 5, edge pulses are distinctly different within the range of $5^{\circ} \mathrm{C}$ and intensity detecting is reliable. On the other hand, the curve of temperature-spectrum width from the common FBG is good within a wide temperature range, but affected by the crosstalk of sidelopes, there is fluctuating or even obvious missed orders in the reflective spectrums. At this time, the error from the decision device may be serious. So it is meaningful to optimize apodization according to the measuring range and accuracy of the actual FBG system.

Spectrum width is obtained by direct intensity detecting in this demodulation, and at the same time some temperature information is collected on different points of FBG. There are obvious advantages of measuring nonuniform temperature fields. By analyzing variations of pulse intensity, the gradient will be obtained for monotonic temperature fields. For complex temperature fields, the direction change will be demodulated by comprehensive analysis of reflective spectrums ${ }^{[6]}$.

\section{Conclusions}

In conclusion, the demodulation method of temperaturespectrum width is feasible and the measuring accuracy of temperature is better than $1{ }^{\circ} \mathrm{C}$. It provides a new method for measuring temperature fields.

\section{Acknowledgment}

This work was supported in part by Hubei Natural Science foundation (No.2015CFB436); Guangxi Province Water Power Survey and Design Institute entrusting project (SDHZ2014055)

\section{References}

1. Li Lan, Dong Xin-yong, Zhao Chun-liu, Sun Yi-ling, Jin Shang-zhong, Zhang Zai-xuan. Laser

Optoelectronics Prog. 47, 090603 (2010).

2. Z.C.Zhuo, B.S.Ham. Opt.FiberTechnol.15, 442(2009).

3. Q.Wu, Semenova Y.Opt. Laser Technol.42, 653(2010)

4. Li Bin, TAN Wei-zhong, Liu Yan, Wang Yan-hua, Ren Wen-hua, Jian Shui-sheng1, ActaOpt. Sin. 27, 2133(2007).

5. Tsai P, Fengguo Sun, Gaozhi-xiao, Zhiyi Zhang, Rahimi S, Dayan Ban. Photonic Tech. Lett. 20,300(2008).

6. ZHANG Zi-jia. Sci. Press China. 2009. (in Chinese)

7. Che Ya-liang, Luo Kai-bin, Du Ting-long. ACTA
OPT. SINICA. 29, 2973(2009). (in Chinese)

8. LI Zhi-quan, CAO ping, REN Xiao-li, ZHANG Jing-ru, WANG Yan-nan. J. Optoelectronics-Laser. 22,491(2011).

9. YANG Dong-Zhou, FENG De-Jun, WANG Jing, JIANG Ming-shun, SUI Qimei.J.OptoelectronicsLaser. 20, 1438(2009). (in Chinese)

10. LI Yong-guo, DAI Heng, YAO Kai-fang, WU an, ZHANG Dong-sheng.J. WUHAN UNIV. TECHNOL. 32, 59(2010). (in Chinese)

11. LV Shuang-ming. Shandong Univ.2009. (In Chinese).

12. HAN Ping, ZHOU Zu-de, CHENG Song-lin.J. Optoelectronics Laser. 21,817(2010). (in Chinese)

13. CAI Hong, WEI Zhi-fen. J.Shanxi Univ. (Nat. Sci. ED.).34,237(2011). (in Chinese)

14. YANG Lin, DUAN Kai-Liang, LUO Shi-long, ZHAO Wei. HIGH POWER LASER PART. BEAMS. 23,308(2011). (in Chinese)

15. ZHANG Hao, CHENG Shu-ming. J. Changzhi Univ.27, 4(2010). (in Chinese) 